\title{
Improving the Energy Resolution of Energy Dispersive Spectrometers(EDS) Using Richardson-Lucy Deconvolution
}

Nicolas Brodusch and Raynald Gauvin

McGill University, Montreal, Quebec, Canada

Energy dispersive spectrometers (EDS) are essential tools in combination to scanning (SEM) and transmission (TEM) electron microscopes to characterize a material and determine its chemical composition. However, these detectors suffer of low energy resolution in comparison to other $\mathrm{x}$-ray techniques like wavelength dispersive spectrometers (WDS) [1] or soft x-ray spectrometers [2] which provide energy resolution at the scale of the electron volt $(\mathrm{eV})$. Although well suited for measuring the composition of the sample, EDS efficacity is impeded by $\mathrm{x}$-ray peaks overlaps and is unable to provide information on the bond state of the emitting atoms like the two other x-ray techniques previously mentioned.

In this work, we'll present how the deconvolution of the EDS spectrum using the Richardson-Lucy (RL) algorithm can help providing higher energy resolution as historically reported for electron energy-loss spectroscopy (EELS). This algorithm permits to remove the contribution of the point spread function (PSF) of the spectrometer from the experimental spectrum. In EELS, the PSF is taken as the zero-loss peak of the spectrometer but for EDS, the noise peak (or zero peak), which is the expression of the shaping factor of the spectrometer, can be used as the PSF. EDS deconvolution was previously reported by Watanabe and Williams but their conclusions were mitigated since only limited gain of resolution was obtained at low energies [3].

To evaluate the true gain of RL deconvolution for the EDS analysis, the method was tested on synthetic low energy overlapping peaks using Gaussian shaping with the full width at half maximum (FWHM) following the shaping factor of the spectrometer. Figure 1 shows the effect of the number of iterations on the deconvoluted spectrum between The $\mathrm{Li} \mathrm{K}$ and the noise peaks. The main resolution gains were obtained mainly after a few tens of iterations and Figure $1 \mathrm{~b}$ shows that a possible FWHM reduction of around three is realistic.

However, since the shaping factor of the spectrometer increases with the peak energy, using the noise peak as the PSF does not allow a significant gain of resolution at larger energies. In this case, it is better to use as the PSF a synthetic noise peak where the FWHM is calculated using the broadening function of the spectrometer. Figure $2 \mathrm{~b}$ shows the gain of resolution using RL deconvolution with a broadened noise peak for an energy of $0.5 \mathrm{keV}$ as the PSF on a lithium titanate sample compared to using only the noise peak as the PSF (Figure 2a). The separation of the Ti L $\alpha$, Ti L $\beta$ and O K $\alpha$ peaks is greatly improved and the background is significantly reduced allowing a more effective background fitting. In this presentation, the impact of the deconvolution on the quantification will be exposed in more details and realistic examples will be shown. 

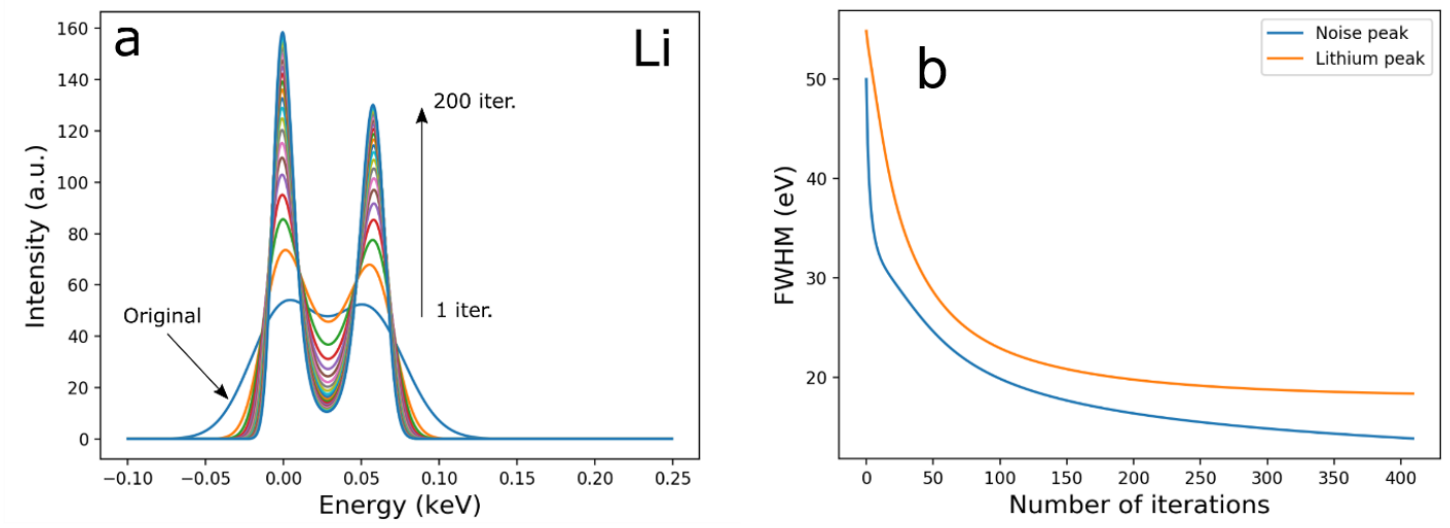

Figure 1. a) Synthetic deconvoluted EDS spectrum with gaussian shaping at increasing iteration numbers (1 to 200 iterations) with the RL algorithm; b) Noise and Li K peaks FWHM as a function of the RL deconvolution iteration number.

a

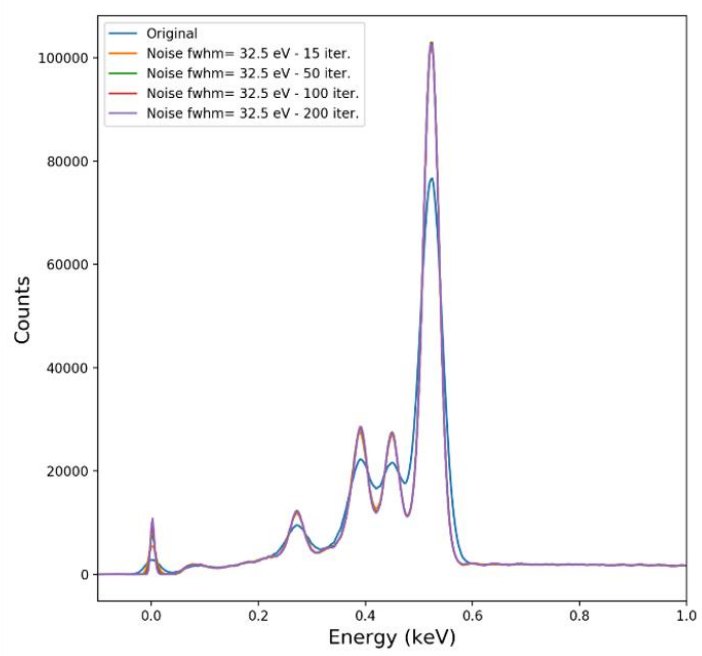

b

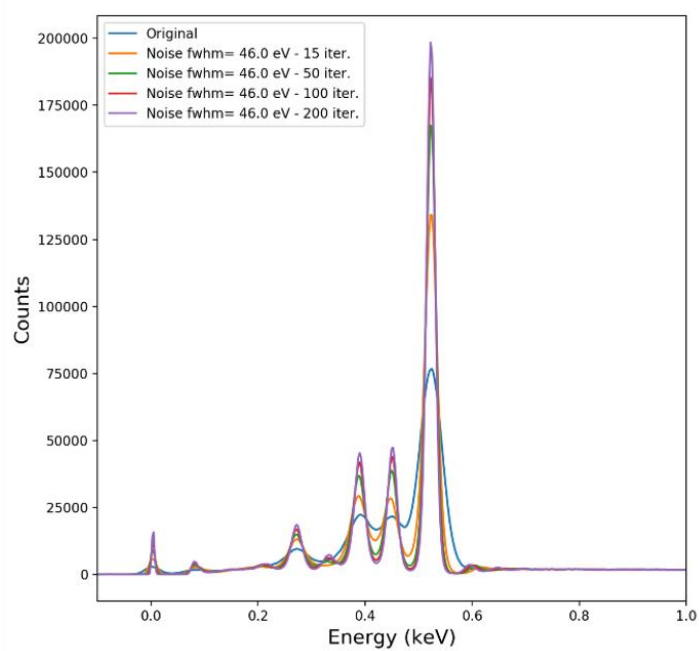

Figure 2. Deconvoluted spectrum from lithium titanate with 15, 50, 100 and 200 iterations with the PSF taken as a) the spectrometer noise peak in vacuum and b) a synthetic computed broadening peak accounting for the broadening function of the spectrometer at energies around $0.5 \mathrm{keV}$.

\section{References}

[1] L. Reimer, Scanning Electron Microscopy: Physics of Image Formation and Microanalysis, Second ed., Springer, 1998.

[2] M. Terauchi, M. Koike, A new high energy-resolution soft-X-ray spectrometer for a transmission electron microscope, Microscopy and Microanalysis, 9 (2003) 894-895.

[3] M. Watanabe, D.B. Williams, Improvements to Energy Resolution of an X-ray Energy Dispersive Spectrum by Deconvolution Using the Zero Strobe Peak, Microscopy and Microanalysis, 9 (2003) 124125. 